\title{
Solute Carrier Family 22 Member 3
}

National Cancer Institute

\section{Source}

National Cancer Institute. Solute Carrier Family 22 Member 3. NCI Thesaurus. Code

C113563.

Solute carrier family 22 member 3 ( $556 \mathrm{aa}, \sim 61 \mathrm{kDa}$ ) is encoded by the human SLC22A3 gene. This protein is involved in mediating membrane potential-dependent transport of org anic cations and may play a role in the disposition of cationic neurotoxins and neurotransmitters in the brain. 